\title{
A Kidney Hydatid cyst with No Conclusive Diagnostic Radiologic Evidence: A Case Report
}

\author{
Amir Nasimfar, ${ }^{1}$ Ebrahim Sadeghi, ${ }^{1,}$ Mohamad Karamyyar, ${ }^{1}$ Ahmad Ali Nikibakhsh, ${ }^{1}$ and Ahad \\ Ghazavi $^{1}$ \\ ${ }^{1}$ Department of Pediatrics, Urmia University of Medical Sciences, Urmia, IR Iran \\ "Corresponding author: Ebrahim Sadeghi, Department of Pediatrics, Urmia University of Medical Sciences, Motaharri Hospital, Kashani Ave, Urmia, IR Iran. Tel: \\ +98-9143492885, Fax: +98-443229320, E-mail: sadeghi.e@umsu.ac.ir
}

Received 2017 August 15; Revised 2017 October 29; Accepted 2017 November 07.

\begin{abstract}
Background: Hydatid disease is a zoonotic parasitic infection that affects humans in many parts of the world, including Iran. It is caused by the larval stage of a tapeworm called Echinococcus. Human is an intermediate host infected accidentally by the ingestion of eggs excreted in the feces of dogs. This infection most commonly involves the liver and lung. Other site localizations are rare including kidney that is seen in $2 \%$ to $4 \%$ of cases.

Case Presentation: Here we report a 9-year-old boy with kidney involvement in a village near Poldasht city, West Azerbaijan, Iran, admitted on Jan 2013. He referred to our hospital with a left upper quadrant (LUQ) mass without any other symptoms, which was discovered by his mother accidentally. Sonography and CT scan with and without contrast revealed large hypodense fluid containing a mass arising from the left kidney that stretched renal parenchyma. Surgery consult was done and radical nephrectomy with left adrenalectomy and retroperitoneal lymphadenectomy due to the suspicion of malignancy was performed. In addition to surgery, he received the first dose of chemotherapy for Wilms tumor and after the pathologic report, albendazole started for him. A definitive presurgery diagnosis is not always possible by radiographic evidence. Therefore, the hydatid disease should be in the differential diagnosis of renal mass. Only surgery and pathologic findings can confirm the diagnosis. In our case, due to the lack of a definitive radiologic evidence for hydatid diagnosis, we did not start albendazole presurgery and albendazole started and continued for six months after the pathologic report confirmed the hydatid disease.
\end{abstract}

Conclusions: We recommend that albendazole starts in endemic areas for abdominal masses with a non-definitive diagnosis.

Keywords: Cyst, Kidney, Wilms Tumor, Echinococcus granulosus

\section{Background}

Hydatid disease is a parasitic infection caused by the larval form of Echinococcus granulosus. It is caused by the larval stage of a tapeworm called Echinococcus. Human is an intermediate host infected accidentally by the ingestion of eggs excreted in the feces of dogs. Three species of Echinococcus can cause hydatid disease, the most common type of which is Echinococcus granulosus, whereas Echinococcus multilocularis and Echinococcus oligartus account for a small number of cases.

Most infected humans are asymptomatic; it might take five to twenty years for a cyst to grow to its symptomatic size (1).

This infection is found worldwide and human Echinococcus granulosus remains a significant health problem for the Middle East and Arabic North African countries including Iran. Dogs have an important role in the transmission of the disease in Iran and the infection rate of Echinococcus granulosus in the definitive host dogs was reported in a range from $5 \%$ to $49 \%$ (2).
Although all localizations are possible, renal hydatid cyst is still rare (3). Hydatid cyst rarely is seen after 40 years of life. Some authors reported the prevalence of kidney hydatid cyst at $1.2 \%$ in children and $2 \%$ in adults $(4,5)$.

Imaging methods, including sonography, computerized tomography (CT), and magnetic resonance imaging (MRI), can provide a definitive differential diagnosis in many cases; but the diagnosis may be problematic in others. In our case, the typical confirmative radiological findings including detached endocyst, "water lily sign," calcification, and daughter cyst were not detected. Serodiagnostic tests based on antibody detection show high sensitivity and advisable specificity(6). For example, eosinophilia, the Casoni (intra-dermal skin) and Weinberg (complement fixation),

Immuno-fluorescence, indirect hemagglutination, and Enzyme-linked immunosorbent assay (ELISA) can be used. ELISA is highly specific to hydatid disease as $98 \%$ (7, 8).

However, there is no serological or immunological test 
pathognomonic for hydatid disease (8).

Treatment for renal Echinococcus is mainly surgical and total or partial nephrectomy is the treatment of choice. In patients with isolated cyst involving kidney in preoperative diagnosis, the retroperitoneal approach is preferred to avoid disseminating the disease into the peritoneal cavity while, in patients with an uncertain preoperative diagnosis, radical nephrectomy should be performed $(8,9)$.

Medical therapy with mebendazole or albendazole is used as prophylaxis postoperatively to prevent recurrences. Preoperative medical therapy should be initiated at least 4 days before the operative procedure. Albendazole is the drug of choice in two daily doses adding up to 10 - 15 $\mathrm{mg} / \mathrm{kg} /$ day (10-12).

\section{Case Presentation}

After obtaining informed consent from the parents, we report a 9-year-old boy who was living in a village near Poldasht city, West Azerbaijan, admitted on Jan 2013. He referred to Mottahari hospital that is a referral center for pediatric and surgery in West Azerbaijan and consists of all subspecialties in pediatrics. The patient presented with a left upper quadrant mass that was discovered by his mother accidentally 10 days before admission.

He had no pain, weight loss, fever, or other symptoms in this period.

In the physical examination, there was a large $\left(10^{*} 12\right.$ $\mathrm{cm})$, soft, non-tender, and slightly mobile mass of LUQ.

Routine blood and urine tests, liver and renal function tests, and electrolytes were normal. In our patient's lab evaluation, eosinophilia was not reported. The serology test was not checked before surgery because of Wilms tumor as a primary diagnosis requiring an emergency diagnostic and therapeutic action.

Sonography as the first imaging technique was recommended that showed a cystic mass with76*78*123 $\mathrm{mm}$ in size full of echo-free fluid with $300 \mathrm{cc}$ volume and a compressive effect on the left kidney. There was no reported evidence of specific finding as detached endocyst inside the cavity, "water lily sign" and calcification. CT scan with and without contrast was requested and showed large hypodense fluid containing mass arising from the left kidney that stretched renal parenchyma. In the CT scan of our patient, no specific finding as daughter cyst in favor of the diagnosis of hydatid cyst was reported (Figure 1).

In the evaluation of other organs such as liver and lung, we did not find any involvement.

Our hospital laboratory devices were calibrated in a regular period with standard criteria settings. In addition, the CT scan device was calibrated regularly according to the manufacturer settings.

Surgery consult was done and radical nephrectomy with left adrenalectomy and retroperitoneal lymphadenectomy due to the suspicion of malignancy was performed. The gross form of the kidney during and after surgery was revealed with abnormal size and shape of the kidney. Left Nephrectomy specimen with large cysts was received with $76^{*} 78 * 123 \mathrm{~cm}$ in diameter. The outer surface was irregular with thinning of the cortex. In addition to surgery, the patient received the first dose of chemotherapy with actinomycin D 75 microgram per kg for a probable diagnosis of Wilms tumor.

After preparation of the pathologic report, the patient was labeled as kidney hydatid disease and chemotherapy regimen discontinued and albendazole and Continued started for six months. During this period, the patient was followed with sonography and CT scan. In that period, there was no evidence of relapse of hydatid mass and albendazole complications (Figure 2).

\section{Discussion}

Hydatid disease is endemic in Iran and remains an ongoing health problem (2).

This infection most commonly involves the liver and lung. Other site localizations are rare such as kidney that is seen in $2 \%$ to $4 \%$ of cases (3-5).

The definite preoperative diagnosis is difficult because it can mimic other diseases such as malignant renal tumors (7). Due to silent and non-specified clinical presentation of renal hydatidosis, it may remain undiagnosed for a prolonged time until presentation as a massive palpable mass $(7,8)$. By using different diagnostic methods such as physical examination, imaging study, and serologic and urine study, only $50 \%$ of cases can be diagnosed preoperatively (7). Like in previous reports, our patient was asymptomatic until the mass was found by his mother incidentally.

The most common sign and symptoms are a palpable mass, flank pain, hematuria, malaise, fever, and hydaturia (9).

Sonography is the first radiologic investigation that is noninvasive, sensitive, available, reliable, and inexpensive $(3,10)$. CT scan can show calcification and daughter cysts easier than sonography does with high sensitivity and accuracy (10). In our case, sonography and CT scan did not show any evidence of daughter cyst, calcification, or other radiologic signs in favor of hydatid cyst diagnosis.

Preoperative medical treatment with albendazole reduces recurrence and sterilizes the cyst wall (11). In our case, due to the lack of evidence of hydatid diagnosis, 

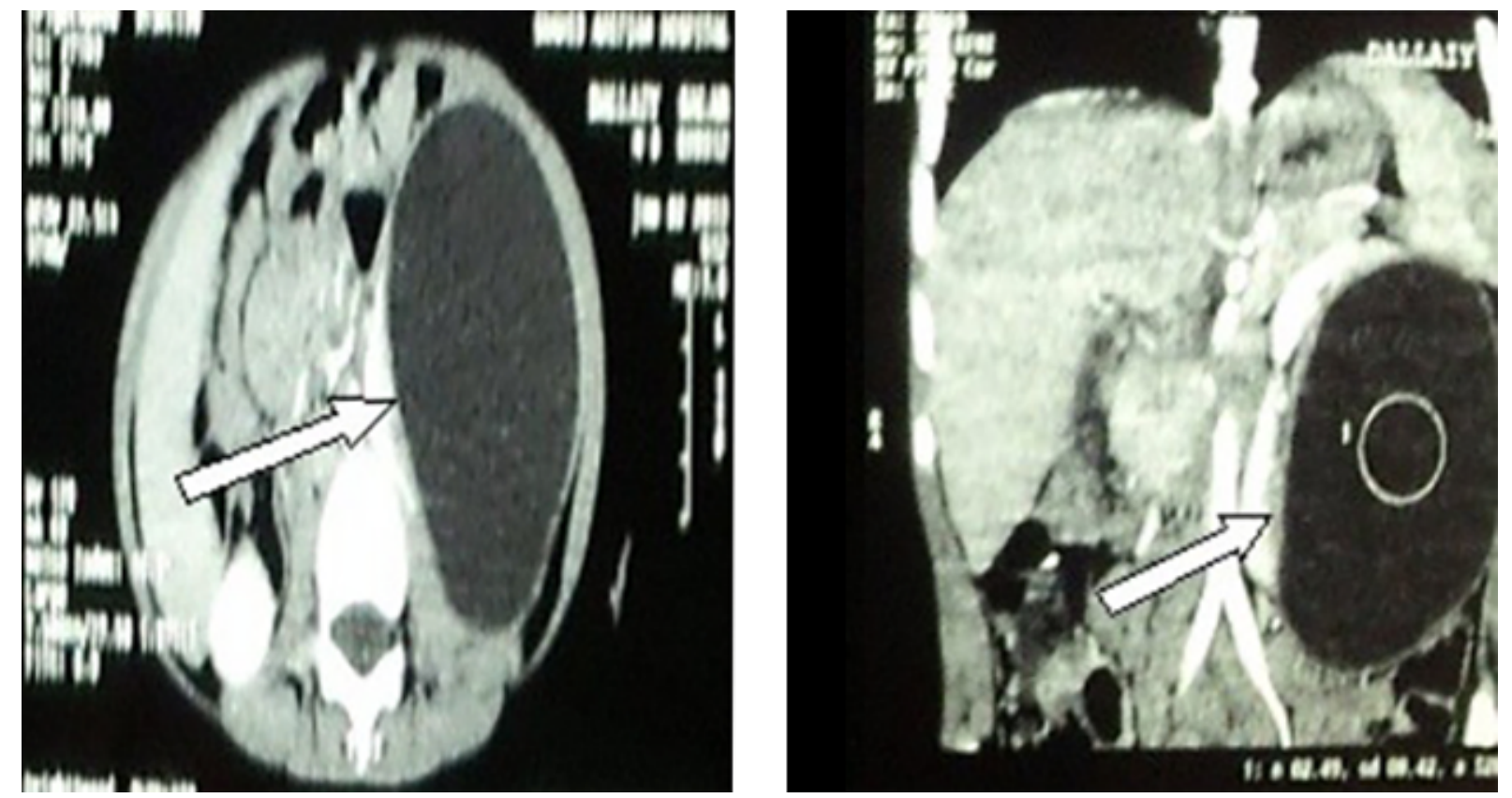

Figure 1. Hypodense fluid containing a mass with $78^{*} 123 \mathrm{~mm}$ in size arising from the left kidney stretching renal parenchyma
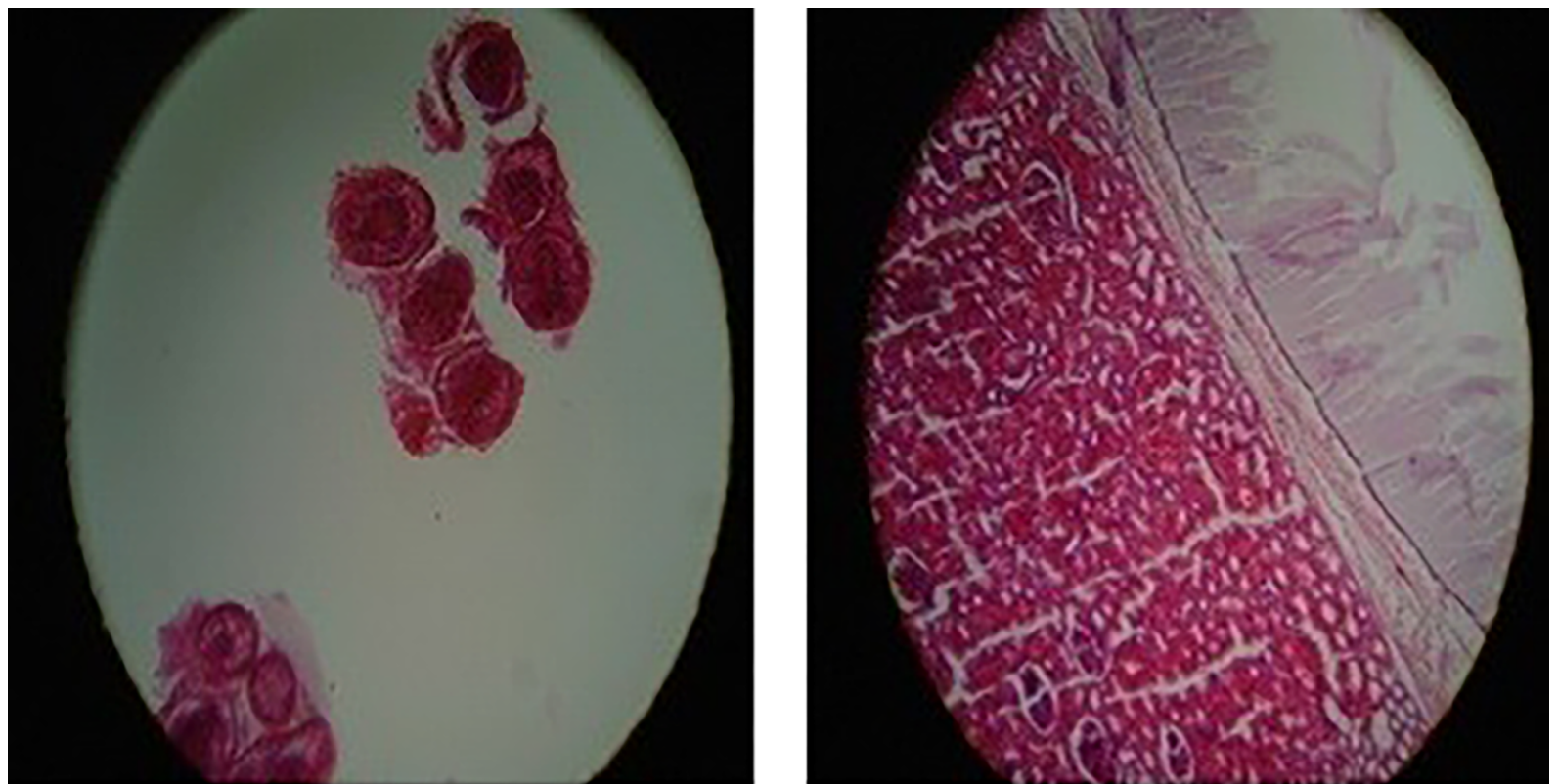

Figure 2. Hydatid cyst of the kidney. Kidney tissue and a cyst with a chitinous layer on the right side of the picture are visible. Hematoxylin and Eosin stain, X10. Histopathological examination of surgically removed Scoleces (left slide). Hematoxylin and Eosin stain, X40

we did not start albendazole presurgery and albendazole started after the pathologic report confirmed the hydatid disease. We continued albendazole for six months and during this period; we followed the patient with sonography and CT scan. In that period, there was no evidence of relapse of hydatid mass and albendazole complications.

A definitive presurgery diagnosis is not always possible by radiographic evidence. Therefore, hydatid disease 
should be in the differential diagnosis of renal mass. Only surgery and pathologic findings can confirm the diagnosis. In our case, due to the lack of definitive radiologic evidence for hydatid diagnosis, we did not start albendazole presurgery and albendazole started after the pathologic report confirmed hydatid disease.

Because in our case report a definitive diagnosis before surgery was not possible and the patient treatment started before surgery with actinomycin D instead of mebendazole, it can be interesting and unique.

\subsection{Conclusions}

A radiologic evidence does not always confirm the diagnosis before surgery. Although the hydatid disease should be considered in renal cystic lesions in endemic areas, only surgery and pathologic findings can confirm the diagnosis. We recommend that albendazole starts in endemic areas for patients with an abdominal mass and nondefinitive diagnosis.

\section{Acknowledgments}

We thank Dr. Mohammad Vali Baluch for his cooperation and excellent surgery.

\section{Footnote}

Conflict of Interests: The authors declare that they have no conflict of interest.

\section{References}

1. Zargar-Shoshtari M, Shadpour P, Robat-Moradi N, Moslemi M. Hydatid cyst of urinary tract: 11 cases at a single center. Urol J. 2007;4(1):41-5. [PubMed: 17514611].

2. Ahmadi NA, Bodi F. Clinical presentation, localization and morphology of hepato-pulmonary hydatid cysts in patients operated in Tehran. World Appl Sci J. 2011;12(9):1544-8.

3. Rami M, Khattala K, ElMadi A, Afifi MA, Bouabddallah Y. The renal hydatid cyst: report on 4 cases. Pan Afr Med J. 2011;8:31. [PubMed: 22121440]. [PubMed Central: PMC3201596].

4. Broughton ME. Hydatid cyst of the kidney in a child. Arch Dis Child. 1957;32(165):466-8. [PubMed: 13479157]. [PubMed Central: PMC2012155].

5. Seetharam V, Khanna V, Jaiprakash P, Kosaraju K, Thomas J, Mukhopadhayay C. Primary hydatid cyst of the kidney and ureter with hydatiduria in a laboratory worker: a case report. Case Rep Nephrol. 2012;2012:596923. doi: 10.1155/2012/596923. [PubMed: 24555138]. [PubMed Central: PMC3914243].

6. Ahmadi N, Hamidi M. Unusual localizations of human hydatid disease in Hamedan province, west of Iran. Helminthologia. 2010;47(2). doi: 10.2478/s11687-010-0015-x.

7. Kalinova K, Usunov N. Primary renal echinococcosis-experience whit 14 cases. JImab. 2007;13(1):5-7.

8. Gogus C, Safak M, Baltaci S, Turkolmez K. Isolated renal hydatidosis: experience with 20 cases. J Urol. 2003;169(1):186-9. doi: 10.1097/01.ju.0000042277.16553.5c. [PubMed: 12478132].

9. Horchani A, Nouira Y, Kbaier I, Attyaoui F, Zribi AS. Hydatid cyst of the kidney. A report of 147 controlled cases. Eur Urol.2000;38(4):461-7. doi: 10.1159/000020325. [PubMed: 11025387].

10. Ameur A, Lezrek M, Boumdin H, Touiti D, Abbar M, Beddouch A. [Hydatid cyst of the kidney based on a series of 34 cases]. Prog Urol. 2002;12(3):409-14. [PubMed: 12189747].

11. Karakoc ZC, Kasimcan MO, Pipia AP, Tore G, Alberti A, Varcasia A, et al A life-threatening brainstem compression by cerebral Echinococcus granulosus. Infez Med. 2016;24(1):62-6. [PubMed: 27031900].

12. Soares AT, Couto C, Cabral MJ, Carmona L, Vieira I. Renal hydatid cyst: medical treatment. J Bras Nefrol. 2016;38(1):123-6. doi: 10.5935/01012800.20160017. [PubMed: 27049373]. 